Widefield ScIENCE AND TeChNOLOGY For the SKA

SKADS CONFERENCE 2009

S.A. Torchinsky, A. van Ardenne, T. van den Brink-Havinga, A.J.J. van Es, A.J. Faulkner (eds.)

4-6 November 2009, Château de Limelette, Belgium

\title{
SKA HI end2end simulation
}

\author{
H.-R. Klöckner ${ }^{1}$, R. Auld ${ }^{2}$, I. Heywood ${ }^{1}$, D. Obreschkow ${ }^{1}$, F. Levrier ${ }^{3}$ and S. Rawlings ${ }^{1}$ \\ ${ }^{1}$ Oxford Astrophysics, Denys Wilkinson Building, Keble Road, Oxford, OX1 3RH, United Kingdom \\ 2 Cardiff University, Queens Building, The Parade, Cardiff, CF24 3AA, United Kindom* \\ ${ }^{3}$ LERMA/LRA -UMR 8112 - Ecole Normale Supérieure, 24 rue Lhomond, 75231 Paris CEDEX 05, France *
}

\begin{abstract}
The current status of the HI simulation efforts is presented, in which a self consistent simulation path is described and basic equations to calculate array sensitivities are given. There is a summary of the SKA Design Study (SKADS) sky simulation and a method for implementing it into the array simulator is presented. A short overview of HI sensitivity requirements is discussed and expected results for a simulated HI survey are presented.
\end{abstract}

\section{Introduction}

One of the key-science goals of the SKA is to detect most of the neutral hydrogen (HI; $1420.4 \mathrm{MHz}$ in the rest frame) content of galaxies out to cosmological redshifts of $\mathrm{z} \sim 1$ (for further details see e.g. Science with the Square Kilometre Array eds. Carilli and Rawlings or Cosmology, Galaxy Formation and Astroparticle Physics on the Pathway to the SKA eds. Klöckner, Rawlings, Jarvis and Taylor). The technical parameters that determine the performance of the SKA have been identified previously during the SKADS program and are defined in the Benchmark scenario (Alexander et al. 2007). At the moment the SKA design includes three distinct telescope technologies in order to cover the required frequency range, i.e. between a few hundreds of $\mathrm{MHz}$ to $10-25 \mathrm{GHz}$. The parameter space one needs to cover to assess the performance of the low-frequency sparse dipole array, the mid-frequency aperature array (AA), or the high-frequency dish array is enormous, and this is impossible to accomplish via a single telescope simulation. To get a basic understanding of the array parameters and their influence on the quality and sensitivity of the final images, one can parameterise each of the different antenna types in terms of a dish equivalent. For a dish array, two basic parameters are the system equivalent flux density (SEFD) and the image sensitivity $(\Delta \mathrm{I})$. Respectively, these are given by

$$
\mathrm{SEFD}=\frac{\mathrm{T}_{\mathrm{sys}} 2 \mathrm{k}}{\eta_{a} \mathrm{~A}},
$$

where $T_{\text {sys }}$ is the system temperature $[\mathrm{K}], \mathrm{k}$ is the Boltzmann constant, A is the collecting area $\left[\mathrm{m}^{2}\right]$, and $\eta_{a}$ is the aperture efficiency, and (for a naturally weighted image) the image sensitivity can be calculated via:

$$
\Delta \mathrm{I}=\frac{\mathrm{SEFD}}{\sqrt{\mathrm{N}(\mathrm{N}-1) \mathrm{N}_{\text {Stokes }} \mathrm{t} \Delta v}},
$$

where $\mathrm{N}$ is the number of Antennas, $\mathrm{N}_{\text {Stokes }}$ is the number of Stokes parameters, $\mathrm{t}$ integration time [s], and $\Delta v$ is the bandwidth [Hz] (Wrobel and Walker 1999). A sensitivity calcula-

\footnotetext{
^ This work was supported by the European Commission Framework Program 6, Project SKADS, Square Kilometre Array Design Studies (SKADS), contract no 011938.
}

tor can be found at www-astro.physics.ox.ac.uk/ $/ \mathrm{hrk} /$ ARRAY_EXPOSURE.html.

In order to increase the image sensitivity both equations dictate that the system temperature and the effective area are crucial to the telescope performance. However in the case of continuum emission that is spectrally well-behaved (spectral index of around zero), the image sensitivity can be increased by trading the SEFD for increased bandwidth. For spectral line observations, where the bandwidth is tailored to the width of the expected signal, this cannot be done. Bandpass stability also plays a crucial role in the image quality in this regime.

In this article we describe an end-to-end (e2e) HI simulation plan that is focused on exploring a more manageable parameter space defined by the system temperature, effective area, and the spatial configuration of the array.

\section{Simulation}

Any aperture synthesis telescope acts as a spatial frequency filter and an analytic determination of the final image quality is in most cases impossible. Therefore the purpose of any interferometry simulation is to define a practical sensitivity limit with respect to the theoretical estimates. Generally one would like to have a full array simulation that simulates the complete signal path from the astronomical source up to and including the receiver electronics. Such an approach is very complicated, computationally expensive and for the purposes of a full SKA simulation completely impractical. The e2e simulation can be broken down into individual components each of which is treated as a standalone simulation. If one is interested in the electromagnetic properties (e.g. the directional gain and its stability) of individual dish designs one needs to take into account the telescope structure and make use of a full electromagnetic simulation (e.g. Holler et al. 2008). Similar simulations are needed if one is interested in the performance of an aperature array and its primary beam pattern (e.g. OSKAR: Dulwich et al. 2010, www. oerc.ox.ac.uk/research/oskar). So far little has been done to simulated the electronic path from the receiver to the correlator, but that is certainly in the scope of the PrepSKA (www.jb.man.ac.uk/prepska) program. Finally, after the correlation stage the data quality and array perfor- 


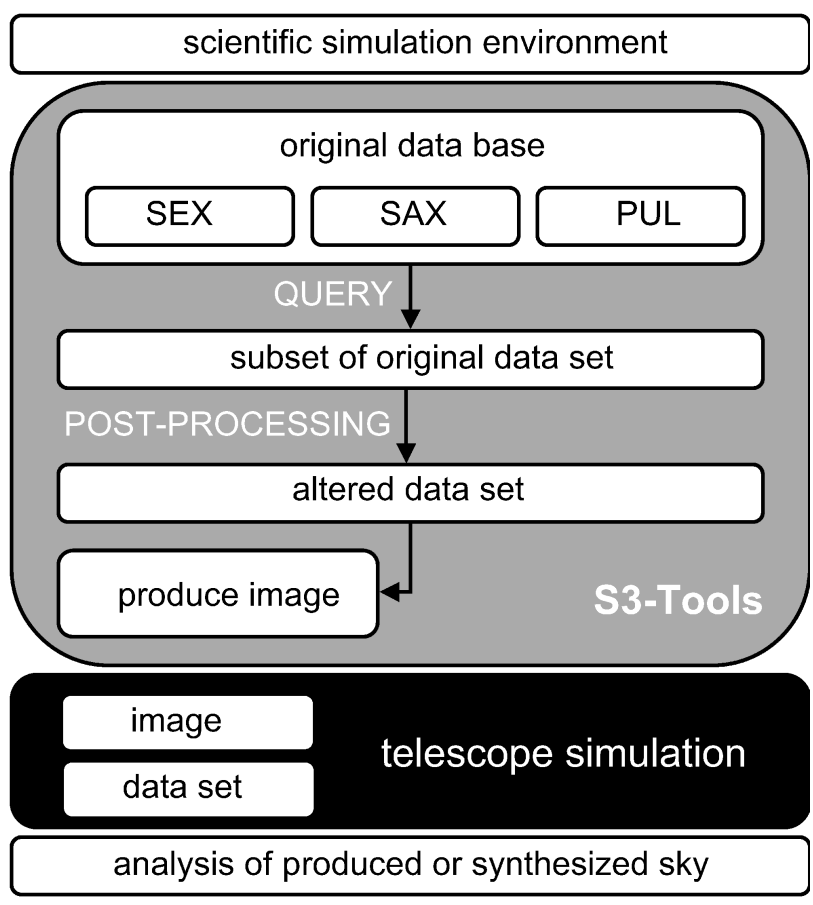

Fig. 1: Overview of a full simulation path.

mance can be evaluated by the simulating the response of individual baselines, or by simulating and assessing the final image.

Radio astronomy software packages which are currently available and offer simulation functionality (e.g. AIPS, CASA, MeqTrees) generally focus on generating a model visibility set given a model sky and a set of parameters which describe the observation. Such functionality has emerged naturally, due to the self-calibration process relying on the generation of model visibilities, however the sophistication of the simulations offered by packages has generally evolved beyond this fundamental role, particularly in the case of MeqTrees.

An overview of the developed e2e simulation path is shown in Fig. 1. The grey box shows the steps we take to generate a model sky. In our case this is based on the SKADS Simulated Skies $\left(\mathrm{S}^{3}\right)$, which are a series of databases describing the properties of a range of simulated astrophysical objects. The databases themselves are discussed further in the next section. Specific subsets of these databases can be retrieved and processed, and finally converted into either a 2-D image or a 3-D datacube (the third axis being frequency) with optional Gaussian noise ${ }^{\mathrm{a}}$. These represent idealised radio skies which can then be fed into a telescope simulation package. The array simulator is represented in Fig. 1 by the black box, which will take the model sky image and generate a model data set based on this. The final step is the analysis of this end product. In the case of the e2e HI simulation the analysis step involves run-

\footnotetext{
a A suite of Python-based routines with user-friendly GUIs, collectively known as the S3-Tools allows access, manipulation and imaging of the SKADS Simulated Skies. These tools have been developed by F. Levrier and a general overview can be found in this volume (Levrier, 2010 in these proceedings).
}

ning both the 'idealised' and 'observed' HI datacubes through a source finder algorithm and comparing the two resulting catalogues. This comparison can then be used to benchmark a specific telescope design or observing strategy. In addition, the ability of the $\mathrm{S}^{3}$-tools to produce maps with Gaussian noise allows to test the source finding algorithm and provide measures for completeness studies.

\subsection{The sky simulation}

Here we present a short summary of the sky simulations that can be used within the array simulator. Further details on the simulations together with a webform which can be used to query the databases is available on the Oxford $\mathrm{S}^{3}$ webpage $^{\mathrm{b}}$. The full suite of simulations is presented using two distinct products. Properties of individual extragalactic objects and of Galactic pulsars are stored in databases (SEX, SAX, PUL), whereas morphologically complex structures such as the Global Sky Model (GSM) and the signals of the Epoch of Reionization (EOR) are available as images.

The GSM is the radio foreground of our Galaxy which has been modeled by the radio and (sub)- millimeter emist sion between $10 \mathrm{MHz}$ to $100 \mathrm{GHz}$. In addition to the diffuse Galactic emission it also includes emission from individual point sources e.g. supernova remnants (de Oliveira-Costa et al. 2008, space.mit. edu/home/angelica/gsm).

The EOR images display the HI line signal of the Intergalactic medium (IGM) during the Epoch of Reionization. This simulation covers the redshift range between $\mathrm{z}=5.6$ and $\mathrm{z}=23.6$. In addition to the ionization field, the effect of inhomogeneous heating of the IGM by X-rays and the Lyman- $\alpha$ radiation field are taken into account. The simulations have been produced in a cubic simulation box with a side length of $\mathrm{s}_{b o x}$ $=100 \mathrm{Mpc} / \mathrm{h}$ and a particle mass resolution of $3 \times 10^{6} / \mathrm{h}$ solar masses (Santos et al. 2008).

The semi-empirical simulation of extragalactic sources (SEX) describes the radio continuum emission in a sky area of $20 \times 20 \mathrm{deg}^{2}$ out to a cosmological redshift of $z=20$. As the name suggests, the sources were drawn from observed (or extrapolated) luminosity functions and grafted onto an underlying dark matter density field with biases which reflect their measured large-scale clustering. This approach puts an emphasis on modelling the large-scale cosmological distribution of radio sources rather than the internal structure of individual galaxies.

Five source types of radio sources have been included in the simulation:

- Radio-quiet AGN [1 core; 36,132,566 sources]

- Radio-loud AGN of the FRI class [1 core + 2 lobes; 23,853,132 sources]

- Radio-loud AGN of the FRII class [1 core + 2 lobes +2 hot-spots; 2,345 sources]

- Quiescent star-forming galaxies [1 disk; 207,814,522 sources]

- Starbursting galaxies [1 disk; 7,267,382 sources]

\footnotetext{
b s-cubed.physics.ox.ac.uk
} 
For each of the source types, the database provides the radio fluxes at observer frequencies at $151 \mathrm{MHz}, 610 \mathrm{MHz}, 1.4 \mathrm{GHz}$, $4.86 \mathrm{GHz}$ and $18 \mathrm{GHz}$, down to flux density limits of $10 \mathrm{nJy}$. Intermediate frequencies can be determined by using the S3tools. In addition to the continuum emission this simulation provides a rough estimate of the HI mass of the starbursting and star-forming galaxies (Wilman et al. 2008). A secound version of these simulations extending the simulated properties into the far-infrared (principally for comparison with data from the Herschel satellite) has now been completed (Wilman et al. 2010).

The semi-analytic simulation (SAX) provides the properties of neutral atomic $(\mathrm{HI})$ and molecular $\left(\mathrm{H}_{2}\right)$ hydrogen in galaxies and associated radio and sub-millimeter emission lines, i.e. the HI-line and various CO transition lines. This simulation relies on the Millenium simulation of cosmic structure (Springel et al. 2005), which reliably recovers comoving length scales from $10 \mathrm{kpc}$ to several hundred $\mathrm{Mpc}$ and galaxies with cold hydrogen masses $(\mathrm{HI}+\mathrm{H} 2)$ above $10^{8} \mathrm{M}_{\odot}$.

There are two versions of the SAX database, reflecting the different versions of the Millenium simulation: the full Millenium simulation $\left(\mathrm{s}_{b o x}=500 / \mathrm{h} \mathrm{Mpc} ; \sim 685 \mathrm{Mpc}\right)$ and the smaller test version, called the Milli-Millenium simulation $\left(\mathrm{s}_{\text {box }}=62.5 / \mathrm{h} \mathrm{Mpc} ; \sim 85.6 \mathrm{Mpc}\right)$ where $\mathrm{s}_{\text {box }}$ defines the diameter of the simulated box. Both of the SAX databases have been produced by constructing a mock observing cone from the corresponding simulation box. The opening angle or the field of view (FoV) of these simulations therefore depends on the values of the maximum redshift one requires $\left(\mathrm{z}_{\text {max }}\right)$, e.g. for a redshift of 1 the FoV of the simulation would be $12 \times 12 \mathrm{deg}^{2}$. More information about the FoV of the simulation can be obtained via the simulation page. Currently, radio continuum data is not available, although efforts are being made to add this information (Obreschkow et al. 2009a; Obreschkow et al. 2009b; Obreschkow et al. 2009c)

S3-Tools is used to build mock radio maps or cubes in which the radio emission of the extragalactic radio sources could be combined with the diffuse radio emission of the GSM or EOR.

\subsection{Array simulation}

The developed array simulator ${ }^{c}$ is based on "classical" AIPS and ParselTongue (Greisen 1990; Kettenis et al. 2006), the Python interface to AIPS. The core function of the array simulator makes use of the AIPS task UVCON. The basic input of this task is a list of antenna locations, together with properties of each antenna, such as diameter, system temperatures and aperture efficiencies. Additional inputs are the total time of observation, the integration time per visibility and the input sky model which also defines the pointing position on the sky. The output is a standard UV-FITS data file in which the visibilities correspond to the input model with added Gaussian noise appropriate for the specified antenna characteristics. Dirty or deconvolved images can be produced by invoking the task IMAGR.

\footnotetext{
c A copy of this simulator can be downloaded via wwW-astro. physics.ox.ac.uk/ hrk
}

If the input model consists of a cube instead of a 2-D image then two different simulation paths can be used to generate the "observed sky". If the output map is to be a continuum image formed at the central observing frequency, the visibilities are produced per frequency step and are finally merged into a single visibility set. If instead the output should be a 3-D datacube, the simulation produces a unique visibility set at each frequency step, each of which is then imaged independently. Each plane is finally combined into a cube by using the task MCUBE. For cases where the duration of the observation equals or exceeds that necessary for a full UV coverage, the visibilities are generated for a full synthesis and the noise scaled down accordingly. This cuts down on both processing overheads and the size of the resulting UV data files.

This style of simulation is well suited to the investigation of the completeness of surveys as well as to the understanding of the imaging capabilities when observing individual galaxies. In particular one can investigate the quality of snapshot observations for different array layouts. There are, however, several AIPS-based limitations which have a direct influence on the questions we can ask, and more complicated simulations are needed to address these. Currently the number of array elements which AIPS can handle is limited to 255 (E. Greisen 2009 , private communication), which is sufficient for most of the current arrays but is not enough for a full simulation of, $\Omega$ for example, the LOFAR array at the level of individual dipole elements. One can circumvent this to some extent by combining several elements into a single station. This is relevant since LOFAR does indeed perform correlations between the beamformed data from each station, and not between individual dipoles. This is a model which the SKA is likely to follow in order to minimize the data stream.

Two properties of a real interferometric array which are absent from this simulation framework are primary beam effects and bandpass variability. These are two factors which significantly affect the dynamic range and image quality of a real observation, particularly the former in the case of an aperture array.

Despite the various limitations we feel that AIPS is likely to be the most tested software package and our approach provides a basic simulation pipeline which can provide a reliable check for more complicated simulation efforts (e.g. within MeqTrees or CASA).

In the redshift range around $\mathrm{z}=1$ the SKA's mapping speed has the potential to be revolutionized by mid-frequency aperture arrays (AA). As mentioned before, the simulation software is limited to 255 antennas and it is necessary to combine the aperture arrays into individual stations. The following equation shows how to calculate the total area of an aperture array, assuming Hertzian dipoles, and therefore the equivalent dish diameter of an AA station:

$$
\mathrm{A}_{\mathrm{AA}}=\mathrm{N}_{\text {tile }} \mathrm{N}_{\text {dipole }} \frac{3}{8 \pi}\left(\frac{\mathrm{c}}{v}\right)^{2} \mathrm{~N}_{\text {stokes }}
$$

where $\mathrm{N}_{\text {tile }}$ is the number of tiles and $\mathrm{N}_{\text {dipole }}$ is the number of dipoles per tile. Assuming a square kilometre of collecting area the diameter of a SKA AA station would be $56 \mathrm{~m}$. Note that an aperture efficiency of $80 \%$ and a total of 255 
stations has been assumed for a $\mathrm{S}^{3}$ default SKA realization $\left(\mathrm{S}_{\text {real }}^{3}\right)$. This value only corresponds to observations towards the zenith, and the effective station area varies with elevation. However simulations to investigate the performance of such a simplified AA are still valuable because the layout of the array will directly affect the synthesized beam and therefore the imaging capability. Figure 3 shows a cut through the dirty beam pattern showing a high sidelobe pattern which can be minimized by varying the station distribution within the array configuration (e.g. AntConfig can be used for this purpose; www.kat.ac.za/public/wiki/AntConfig).

The current design of the aperture array has two layouts that have the same core, but differ in the outer regions (R. Bolton 2009 , private communication). The core has a radius of $2.5 \mathrm{~km}$ and has 165 randomly placed stations which are separated at least by $96 \mathrm{~m}$.

- The "concentrated" layout has 72 stations beyond the core placed in 5 spiral arms out to $10 \mathrm{~km}$ (radius). Beyond this 13 stations are placed on the same spiral arms out to $180 \mathrm{~km}$ $\left(\mathrm{S}_{\text {real }}^{3} \mathrm{C}\right)$.

- The "not-concentrated" layout has 85 stations beyond the core logarithmically placed in 5 spiral arms out to $180 \mathrm{~km}$ $\left(\mathrm{S}_{\text {real }}^{3} \mathrm{NC}\right)$.

Figure 2 shows the UV coverage of a test simulation of 1 hour duration. For displaying purposes each visibility has an integration time of 10 minutes. The high density of visibilities and the filled central core will provide high sensitivity and image fidelity when observing diffuse HI emission. However deconvolution of such structure will be difficult because of the dirty beam pattern shown in Fig. 3. The broad sidelobe pattern will add ambiguities during attempts to recover diffuse, extended HI emission.

A final limitation of the simulation pipeline arises at the imaging stage. The images that can be handled by AIPS are limited to 8096 pixels per dimension. For the current SKA layout the maximum baseline length of $360 \mathrm{~km}$ would provide sub-arcsec angular resolution at frequencies higher than $200 \mathrm{MHz}$. Such resolution limits the field-of-view that can be simulated, and producing a simulation covering several square degrees requires the sky to be divided into sub-patches. For example, the spatial resolution of the array configuration at $700 \mathrm{MHz}$ is $0.3 \mathrm{arcsec}$. If we Nyquist-sample the sky then these images need to have a pixel resolution of about $0.1 \mathrm{arcsec}$ per pixel. Taking the AIPS limitation into account, such images would cover a sky area of $0.67 \times 0.67 \mathrm{deg}^{2}$ and thus to simulate a $4 \mathrm{deg}^{2}$ field 9 sub-images are necessary.

\subsection{The $\mathrm{HI}$ e2e simulation}

The anticipated HI simulation will make use of the SAX simulated sky. These simulations do not include a physical model of the associated continuum emission. However, to evaluate the influence of continuum emission to the HI simulation a mock continuum component may be added to the line emission (using the task UVMOD). The HI emission of each galaxy has been pasted into the model cube by using S3-tools, selecting the

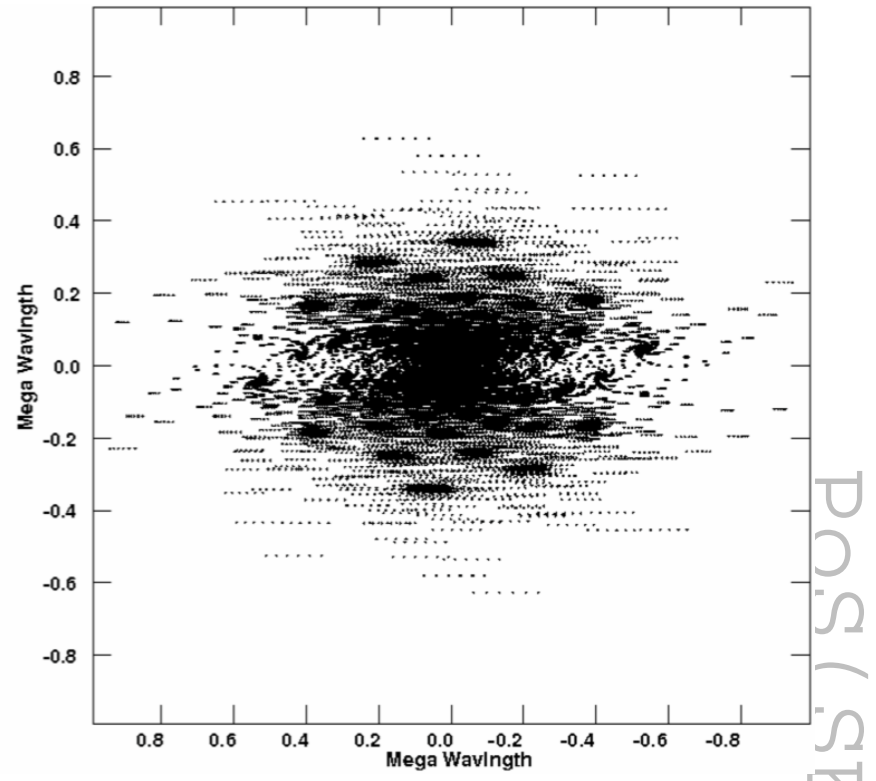

Fig. 2: The UV coverage of the "concentrated" aperture-array layout $\left(\mathrm{S}_{\text {real }}^{3} \mathrm{C}\right)$. The simulation is based on $1 \mathrm{hr}$ integration with an integration time of 10 minutes per visibility. Note that the 10 minutes integration per visibility is for displaying purposes only.

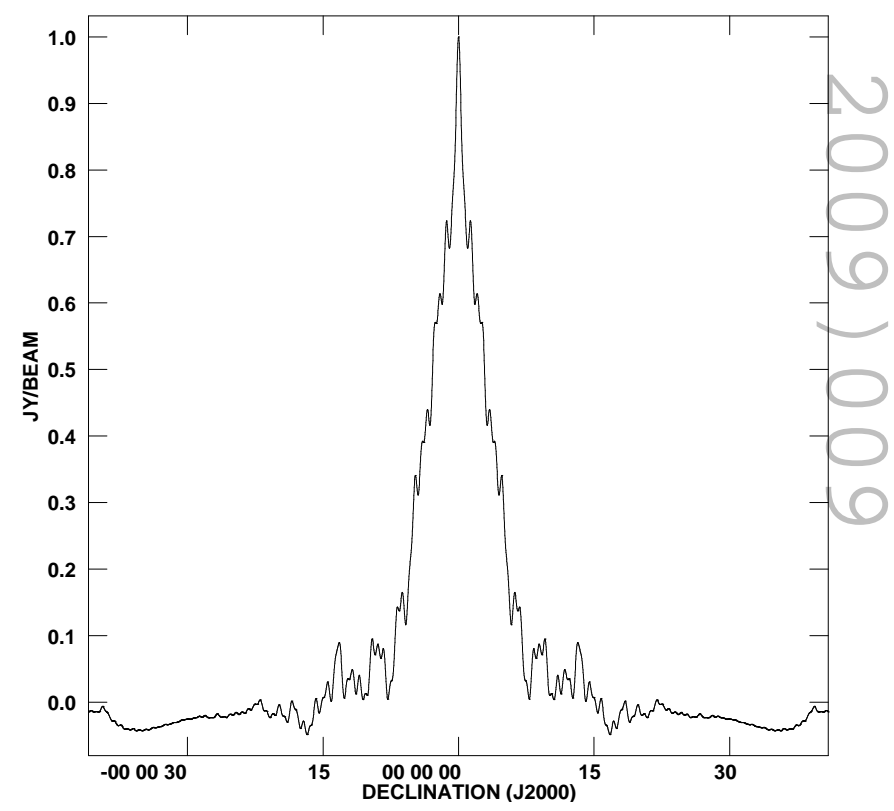

Fig. 3: Cut through the synthesized beam $\left(\mathrm{S}_{\text {real }}^{3} \mathrm{C}\right)$. Due to the high density of stations in the core area, the synthesizes beam profile plateaus around 30 arcsec and shows strong sidelobes at a level of $\sim 10 \%$.

"Oxford" HI templates in the map making tool. The following equation is given to provide a general understanding of the detectability of HI. The basic relationship between HI mass and HI line flux density is defined by

$$
M_{\mathrm{HI}}=\frac{2.36 \times 10^{5}}{(z+1)} \mathrm{D}_{\mathrm{L}}^{2} \frac{\pi}{\sqrt{2 \ln (2)}} \mathrm{S}_{\mathrm{p}} \Delta \mathrm{V},
$$




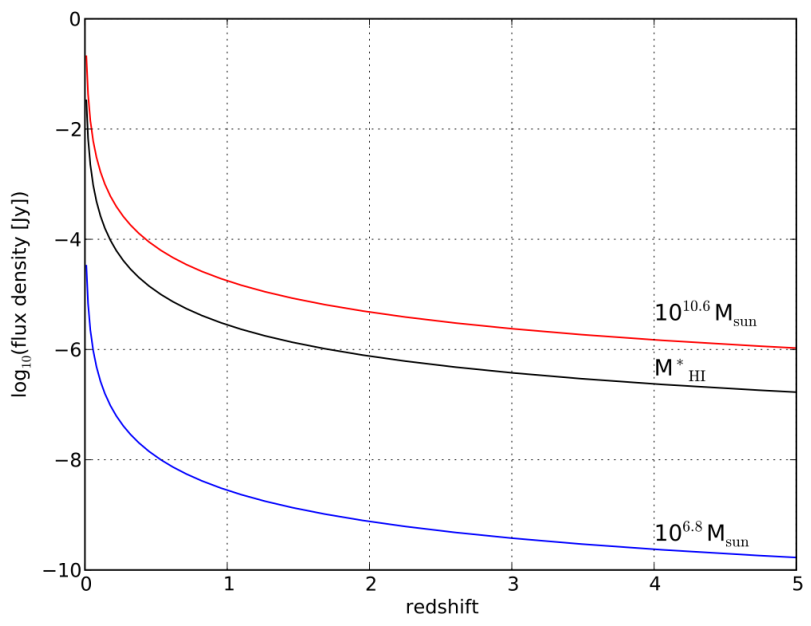

Fig. 4: Flux density of $\mathrm{HI}$ masses with a fixed line width of $164 \mathrm{kms}^{-1}$ versus redshift. The three lines show the entire HI mass range of the HIPASS survey. Note for detection arguments one needs to assume a channel resolution similar to the $\mathrm{HI}$ line width, otherwise the sensitivity is reduced via Eq. 2.

where $\mathrm{D}_{\mathrm{L}}$ is the luminosity distance $[\mathrm{Mpc}]$, assuming a Gaussian line profile where $S_{\mathrm{p}}$ is the peak flux density [Jy] and $\Delta \mathrm{V}$ is the full line width measured at half-maximum [FWHM; $\mathrm{kms}^{-1}$ ]. To investigate how much of the HI mass function one can trace with the SKA, the expected HI flux density is shown in Fig. 4. The HI flux density has been calculated by using average values over the HIPASS sample for the HI line width (FWHM; $164 \mathrm{kms}^{-1}$ ) and for the HI masses (range between $2 \cdot 10^{7}-6 \cdot 10^{10} \mathrm{M}_{\odot}$ ) (Zwaan et al. 2003, 2005). Note that in Fig. 4 to detect the $\mathrm{HI}$ mass at specific redshift one assumes that the entire HI signal is confined within one channel. For a freely chosen 1-sigma rms noise of $1 \mu \mathrm{Jy}$ one could detect $\mathrm{M}^{*} \mathrm{HI}$ galaxies at the 3-sigma level out to a redshift of 1 (Note that a rms noise of $1 \mu \mathrm{Jy}$ would correspond to an integration time of 36 hours, assuming a channel width of $250 \mathrm{kHz}$ and a $\mathrm{T}_{\text {sys }}$ of $50 \mathrm{~K}$.). This is the anticipated aim of the SKA and the HI simulations need to be able to investigate such a cosmic volume. Furthermore, if there is little evolution in the HI mass function one could expect to detect the most massive HI galaxies up to redshifts of about 4 .

The FoV of the Millenium simulation box corresponds to an area of $116 \times 116 \mathrm{deg}^{2}$ at redshift 0.1 , which shrinks to $5.4 \times 5.4 \mathrm{deg}^{2}$ at redshift 4. At low redshifts a HI simulation would help to investigate potential systematic errors in a measurement of the faint end of the HI mass function. Such a simulation would require around 30276 individual simulation runs, based on the field-of-view limitations per-facet, as discussed above. This is a computationally very expensive exercise, and is only worthwhile once the SKA array configuration has been finalised. For investigating and optimising array configurations it makes more sense to perform smaller scale simulations which still allow us to quantify the performance of the array.

We propose such a HI simulation here, which will partly match the co-moving volume of the HIPASS dataset. This re-
Table 1: Simulation parameters at various redshifts. Bandwidth corresponding to a fixed redshift interval of $\Delta z=0.1$. The fraction of the HIPASS volume to the co-moving volume of 1 square degree and $\Delta z=0.1$ (e.g. at redshift 1 such volume would correspond to $0.0008 \mathrm{Gpc}^{3} \mathrm{deg}^{-2}$ ). The FoV of the SAX sky simulations (values are obtained from s-cubed.physics.ox.ac.uk). Expected HI sources per square degree for a flux limit of $3 \mu \mathrm{Jy}$ (assuming a rms of $1 \mu \mathrm{Jy}$ ). For example to investigate the HIPASS volume at redshift 1 a $4 \times 4$ deg $^{2}$ field must be simulated and need to be split into 36 individual subarray-simulations to handle the AIPS image limitation.

\begin{tabular}{ccccc}
\hline redshift & $\begin{array}{c}\text { bandwidth } \\
{[\mathrm{MHz}]}\end{array}$ & $\begin{array}{c}\frac{\text { HIPASSvol }}{\text { co-volume }} \\
{\left[\mathrm{deg}^{2}\right]}\end{array}$ & $\begin{array}{c}\text { SAXsky } \\
{\left[\mathrm{deg}^{2}\right]}\end{array}$ & $\begin{array}{c}\text { SAXsources } \\
{\left[\mathrm{deg}^{-2}\right]}\end{array}$ \\
\hline 0.5 & 59 & 37 & $21.4 \times 21.4$ & 15136 \\
1 & 34 & 16 & $12.0 \times 12.0$ & 7136 \\
1.5 & 22 & 12 & $9 \times 9$ & 1897 \\
2 & 15 & 11 & $7.5 \times 7.5$ & 816 \\
\hline
\end{tabular}

quires a smaller number of individual simulation runs, and it is thus possible to re-run the simulation several times.

HIPASS is a blind HI radio survey of the sky at declinations southwards of 25 degrees. However to calculate the comoving volume we use the initially presented catalogue covering the hole southern hemisphere. The survey covers a sky area of $21314 \mathrm{deg}^{2}$, a redshift range of $\mathrm{z}=0.001-0.042$ (corresponding to a co-moving volume of $0.013 \mathrm{Gpc}^{3}$ ), and has a sensitivity limit approximately corresponding to a peak flux density of $0.05 \mathrm{Jy}$ (see Zwaan et al. 2003, for a full description of the sample completeness). This survey yields a total number of 4315 sources, all of which are subsequently identified with optical galaxies (Doyle et al. 2005).

Using the SAX simulation to mimic the HIPASS survey no extrapolation of the sky simulation is needed because the simulated volume is large enough to cover the entire HIPASS volume. In fact this can been shown by comparing the HIPASS co-moving distance of $171 \mathrm{Mpc}(\mathrm{z}=0.042$ and using the cosmological parameter of the Millenium simulation) which is smaller than the radius of the simulation box, i.e. $500 / 2 \mathrm{Mpc} / \mathrm{h}$ $\sim 342 \mathrm{Mpc}$.

Using the online query of the SAX simulation and defining the redshift and the sensitivity specifications ${ }^{\mathrm{d}}$ of the HIPASS catalogue one obtain 4545 sources. This means that the SAX simulation predicts that the HIPASS catalog contains 4545 sources, which matches the observed number of 4315 sources within $5 \%$. This difference can be partially attributed to the fact that HIPASS yields a continuously varying completeness function rather than a strict peak flux limit. Assuming no flux limits the SAX simulation predicts 772120 source within the HIPASS volume, i.e. 180-times more sources than picked up by HIPASS. However Table 1 shows the expected number counts assuming a minimum peak flux density of $3 \mu \mathrm{Jy}$. The simulation still contain enough source to address e.g. the study of the faint end of the HI mass function and to make statistical significant predictions out to high cosmological distances.

\footnotetext{
d SAX query input: select count(*), from galaxies_line where zapparent between 0.001 and 0.042 and hiintflux*hilumpeak $>0.05$
} 


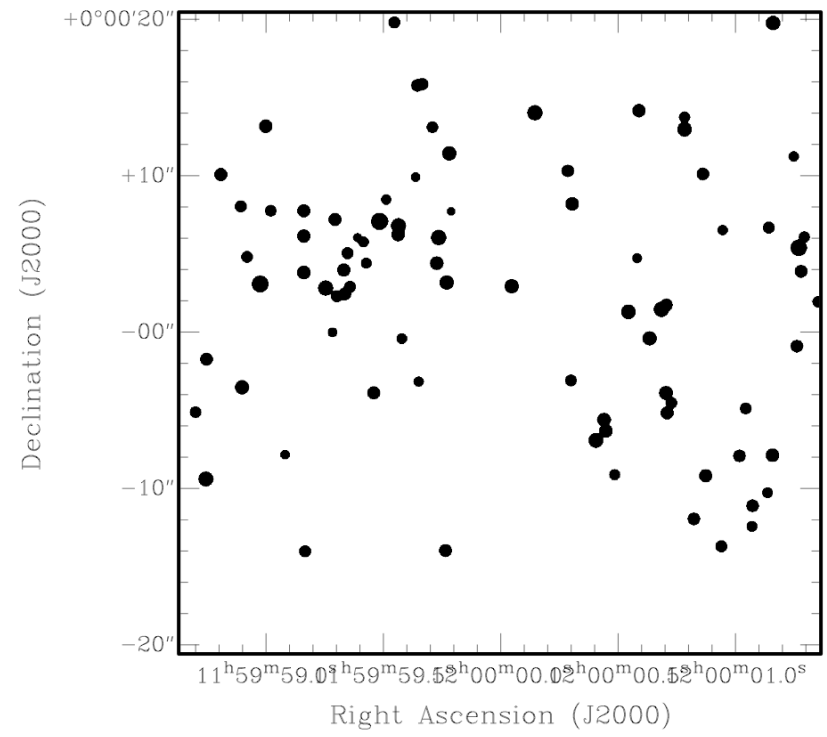

Fig. 5: Example of an HI input sky. The cube has 11 spectral channels covering a frequency range of $11 \times 62.5 \mathrm{kHz}$. For illustration purposes the image shows the channel averaged line emission (using SQASH) and in addition the averaged image has been convolved with a 0.3 arcsec Gaussian beam (using CONVL).

Figure 5 shows the HI intensity map of an input cube. No continuum emission has been added and the individual galaxies are unresolved. The corresponding "observed sky" is shown in Fig. 6. In order to analyse these images the automated source finder, Duchamp e will be used to generate "observed" source catalogues for comparison to the input catalogues. An important part of this analysis will be to produce idealised input images with purely Gaussian noise of an equivalent level to crosscheck the reliability and completeness of the source finding software in the presence of the image artifacts introduced by an interferometer.

\section{Conclusions}

A full e2e simulation path has been developed. The array simulator makes use of images or datacubes based on the $S^{3}$ catalogues to simulate an observation. In the simulations no errors due to calibration or telescope hardware have been introduced, but a simplistic treatment of gain and phase errors could be included at later stages. The analysis of the simulated data sets (either images or cubes) will make use of automated source finder software. In addition to this, a more sophisticated analysis of the simulated data is possible because the simulator produces visibility data as well as images. For example one could investigate the sensitivity of an array to diffuse emission by using the UV-gap $\left(\frac{\Delta \mathrm{U}}{\mathrm{U}}\right)$ analysis techniques (Vir Lal et al. 2009) or by analysing the statistics of Fourier phases (Levrier et al. 2006).

The proposed HI simulations match the requirements for studying the capability of the SKA aperture array when imaging HI structures in nearby galaxies. Such simulations will also

e wWw.atnf.csiro.au/people/Matthew.Whiting/Duchamp

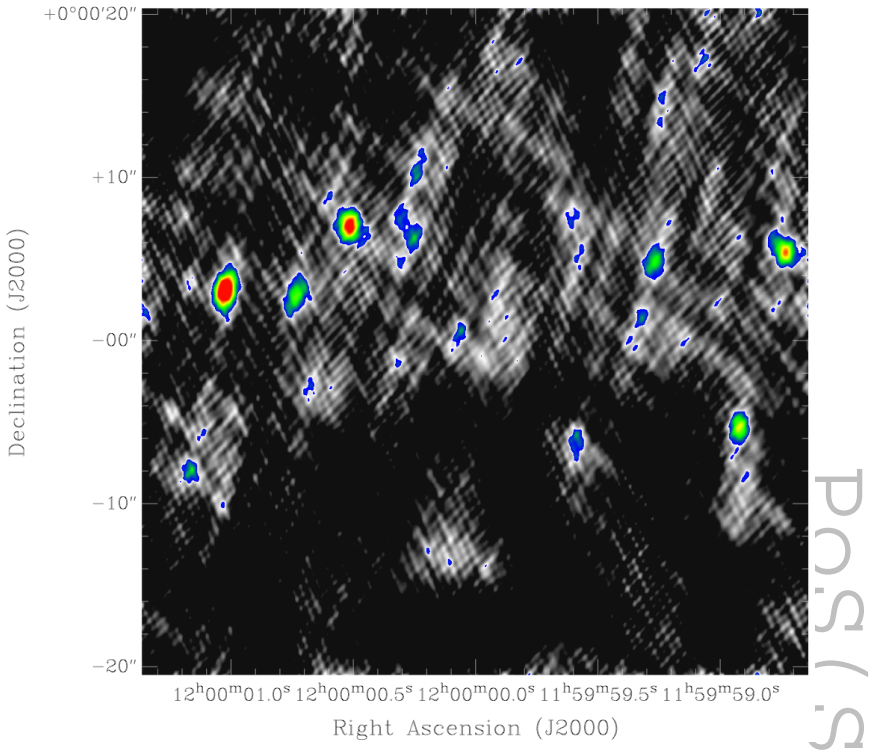

Fig. 6: Example of a simulated HI sky. The rms of this image including sources is of the order of $8 \mu \mathrm{Jy}$. The cleaned image (using 200 clean components) displays the channel averaged line emission.

investigate the impact of different telescope designs on the proposed SKA blind HI surveys. The input HI sky will have an equivalent co-moving volume to that of the HIPASS survey, and this relatively small volume makes it feasible to re-run the simulations with different parameters within a reasonable time. With this setup we are able to analyse the "observed skies" and study the influence that the antenna layout has on a blind $\mathrm{HI}$ galaxy survey by investigating following points:

- completeness (peak flux density)

- positional accuracy

- redshift determination

- necessity of subtracting continuum emission and our ability to do so

Acknowledgements. HRK would like to thank Rosie Bolton for the two array configuration files.

\section{References}

Alexander P., et al., 2007, SKA Memo, 93

de Oliveira-Costa A., Tegmark M., Gaensler B.M., Jonas J., Landecker T.L., Reich P., 2008, MNRAS, 388, 247

Doyle M.T., 2005, MNRAS, 361, 34

Dulwich, F., et al. 2010, in Proc. Wide Field Science and Technology for the SKA, Limelette, Belgium, S.A. Torchinsky et al. (eds), ASTRON, ISBN 978-90805434-5-4

Holler C.M., Hills R.E., Jones M.E., Grainge K., Kaneko T., 2008, MNRAS, 384, 1207

Greisen E., 1990, Acquisition, Processing and Archiving of Astronomical Images, eds. Longo G. and Sedmak G., 125

Kettenis M., van Langevelde H.J., Reynolds C., Cotton B., 2006, ASPC, 351, 497

Levrier F., Falgarone E., Viallefound F., 2006, A\&A,456, 205 
Levrier, F., et al. 2010, in Proc. Wide Field Science and Technology for the SKA, Limelette, Belgium, S.A. Torchinsky et al. (eds), ASTRON, ISBN 978-90805434-5-4

Obreschkow D., Croton D., DeLucia G., Khochfar S., Rawlings S., 2009a, ApJ, 698, 1467

Obreschkow D., Rawlings S., 2009b, ApJ, 696, 129

Obreschkow D., Klöckner H.-R., Heywood I., Levrier F., Rawlings S., 2009c, ApJ, 703, 1890

Santos M., Amblard A., Pritchard J., et al., 2008, ApJ, 689, 1

Springel V., White S.D.M., Jenkins A., et al., 2005, Nature, 435,629

Vir Lal D., Lobanov A.P., Jimenez-Monferrer S., 2009, SKA Memo, 107

Wilman R.J., et al., 2008, MNRAS, 388, 1335

Wilman R.J., Jarvis M.J., Mauch T., Rawlings S., Hickey S., 2010, MNRAS, submitted

Wrobel J.M. and Walker R.C., 1999, Synthesis Imaging in Radio Astronomy II, ASP Conference Series, 180, 171

Zwaan M.A., et al., 2003, AJ, 125, 2842

Zwaan M.A., Meyer M.J., Stavely-Smith L., Webster R.L., 2005, MNRAS, 359, L30 